\title{
A platform for stop flow gradient generation to investigate chemo-
}

taxis

Zuyao Xiao ${ }^{+}$Audrey Nsamela ${ }^{+}$Benjamin Garlan Juliane Simmchen*

Zuyao Xiao, Audrey Nsamela, Dr. Juliane Simmchen

Chair of Physical Chemistry, TU Dresden, 01062 Dreden, Germany

*Email Address: juliane.simmchen@tu-dresden.de

Audrey Nsamela, Dr. Benjamin Garlan

Elvesys SAS, Rue de Charonne 172, 75011 Paris, France

Keywords: Chemotaxis, Colloids, Janus microswimmers, Microfluidics, Stop Flow

The ability of artificial microswimmers to respond to external stimuli and the mechanistical details of their origins belong to the most disputed challenges in interdisciplinary science. Therein, the creation of chemical gradients is technically challenging, because they quickly level out due to diffusion. Inspired by pivotal stopped flow experiments in chemical kinetics, we show that microfluidics gradient generation combined with a pressure feedback loop for precisely controlling the stop of the flows, can enable us to study mechanistical details of chemotaxis of artificial Janus micromotors, based on a catalytic reaction. We find that these copper Janus particles display a chemotactic motion along the concentration gradient in both, positive and negative direction and we demonstrate the mechanical reaction of the particles to small forces deviations, explaining this behaviour.

\section{Introduction}

Starting in the 1940s, stopped flow methods enabled the study of fast chemical kinetics and were thereby pivotal for the understanding of the time dependence in reactions by increasing the time resolution. ${ }^{[1,2]}$ Coupled with spectroscopic techniques, this new strategy quickly dominated the study of enzymatic reactions and influenced the general knowledge on kinetics. Furthermore, stopping the external inflow was quickly found to be a versatile utility and was later adapted to completely different areas of science; to access a variety of shapes in microfluidic synthesis, the Doyle group developed a strategy based on stopping a flow of oligomers before using a shadow mask and UV light to polymerize an array of particles into it: stop flow lithography was born. ${ }^{[3]}$ A few decades after the development of stopped flow techniques, manipulating small volumes of fluids became a research field in itself, coined microfluidics. ${ }^{[4]}$ Developments such as valves, sensors and pressure controllers have, jointly with improved computational facilities and coupling options, expanded the environments that can be created using controlled flows. In particular, the generation of gradients in microfluidics has been of growing interest for the active matter community. ${ }^{[5]}$ Microfluidics was optimized in flowing conditions and stopping the flow was usually slow and frequently led to backflows or circulating media. Depending on the pumping mechanism and the rigidity of the employed materials to create chambers and tubes, this effect can be significant. Therefore, the generation of microfluidic gradients is frequently overlaid by the effects of flowing matter.

Taking a closer look at survival strategies of most living creatures on earth, from bacteria to humans, one of the common traits is migration to search for food. However, at the microscale, and especially in non-equilibrium physical phenomena, many things differ from the macro world; mass and heat transfer are enhanced, viscous forces are dominant over inertial forces, that is by definition a low Reynolds number regime, and thermal fluctuations becomes non negligible. Bacteria are the most studied prokaryotic entities and are often used as model biological microswimmers in the field of active matter. Even though randomness is constantly introduced via Brownian motion and thereby part of the process, bacteria do have the ability to sense and get attracted to certain chemicals: this is called positive chemotaxis. For bacteria, the underlying mechanism is assumed to be strongly based on transduction of sensed signals, ${ }^{[6,7]}$ but there might also be a purely physical contribution. ${ }^{[8]}$ This behavior, jointly with negative chemotaxis, where microorganisms migrate away from a potentially harmful chemical, is found in other types of biological microswimmers and the explanations are as diverse and complex as are these microorganisms.

Also the artificial branch of the field of micro and nanomotors has demonstrated similar tactic behaviors, ${ }^{[9,10,11,12]}$ with chemotaxis being one of the earliest studied principle for self-propelled Janus particles 
and tubular micromotors. ${ }^{[13]}$ To understand how can this attraction to nutrients be mimicked by manmade microswimmers, even though they are deprived of any complex receptors, and to disentangle and model the separate physical contributions, different chemotaxis assays have been created. ${ }^{[14,15]}$ Along with this question come experimental challenges on how to create a controlled and stable gradient in the vicinity of the microswimmers, ${ }^{[16,17]}$ avoiding other parameters, such as flows or capillarity.

Within this work, we have developed a technically refined strategy for gradient generation in microfluidics combined with a stopped-flow technique to perform a chemotaxis assay with artificial Janus microswimmers. The flow based gradient generation using a pressure controller allows for more control over the linear gradient profile, its slope and position of the interface. To precisely stall the flow, a retroactive loop counteracts any backflow by ensuring a net zero pressure difference in the whole system. Removing flows and capillary forces as a variable in our chemotaxis assay ensures a complete observation of the purely physical contributions ${ }^{[18,19]}$ to chemotactic behavior.

\section{Results}

\subsection{Microfluidic gradient generation and stop flow performance}

The microfluidic setup used to generate the gradient and observe the microswimmers behavior in zero flow conditions is illustrated in Figure 1A. The objective of this setup was to instantaneously stop the flow after a gradient was established under constant flow rate in the main chamber of the microfluidic chip. Two microfluidic valves direct the fluids from the outlet to either the waste or to a retroactive loop. During normal flow conditions, the valves (numbered 2 and 3 in the schematic) passed the fluids to the outlet where no pressure is applied. The stop flow procedure consists of switching the valves position to direct the flow into a retroactive loop meeting the inlet lines just ahead of the flow sensors. By applying the same pressure on both inlet reservoirs, the retroactive loops activation ensure a uniform pressure in the whole system, including the microfluidic chamber. The removal of any pressure difference immediately leads to a net zero flow in the system. A description of the automatic sequence is available in Figure S1.

The flow-based concentration gradient is established by enhanced mixing of two fluids joining first in the Y-junction, they are then flowing into a narrow constriction channel that leads to a wider chamber. The mixing time by diffusion $\left(\tau_{D}\right)$ is reduced in the constriction channel because of its smaller width (see equation 1).

$$
\tau_{D} \approx \frac{L^{2}}{2 D}
$$

where $L$ is the characteristic length (here, the channel width) and $D$ the diffusion coefficient of the reagent. The linear gradient generation in the chip and its evolution after stopping flow actuation was calibrated experimentally using a solution of fluorescein in combination with fluorescence imaging over 10 min after stopping the flow (see Figure S2). Adjusting the flow rates allowed to tune the initial slope of the gradient and a constant flow rate of $80 \mathrm{\mu L} / \mathrm{min}$ was set for both inlet fluids in all experiments. Since fluorescein and hydrogen peroxide have different diffusion coefficients, COMSOL simulations were made to model gradient generation and evolution across the chamber more accurately over time (Figure 1B to $\mathrm{E})$. In less than 5 minutes, the slope of the linear gradient is cut by half as the peroxide diffuses towards the less concentrated region. 
A) Flow controller

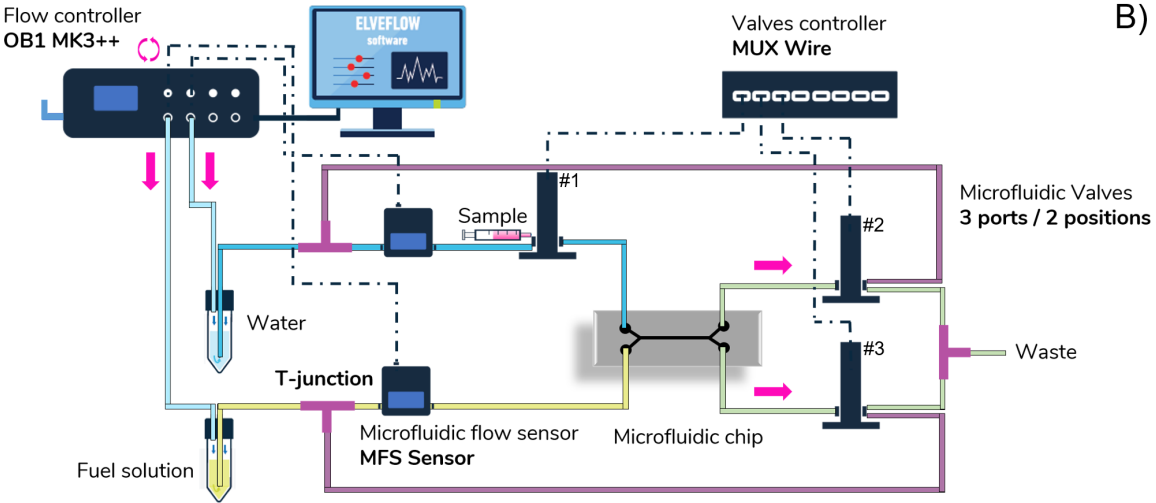

C)

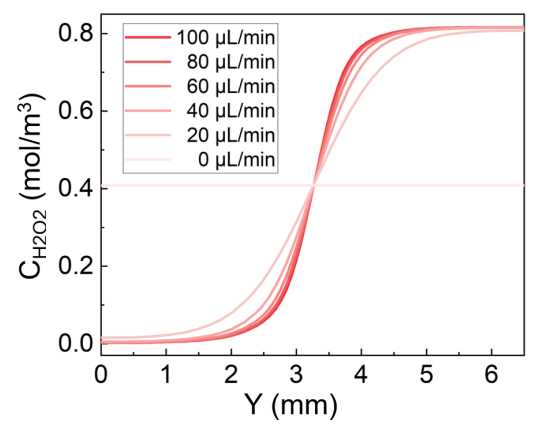

D)

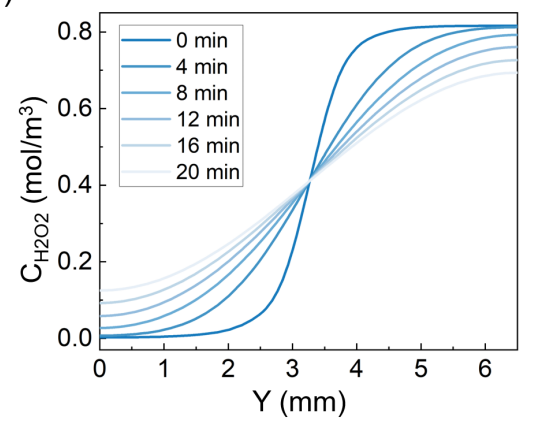

B)
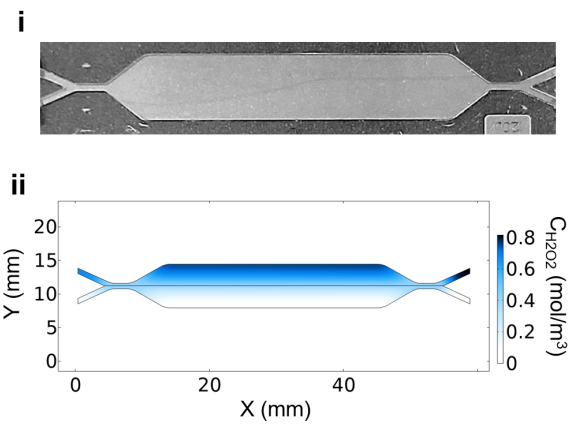

E)

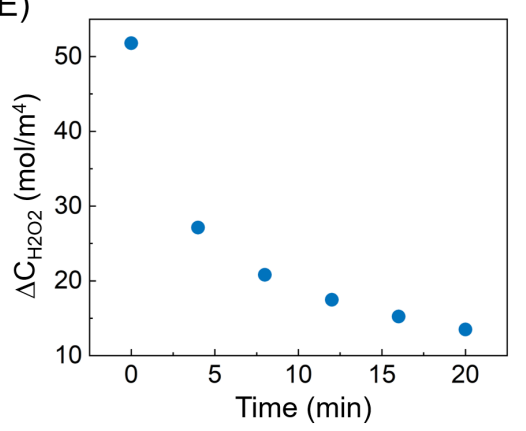

Figure 1: Gradient generation in a microfluidic chip and stop flow performance. (A) Schematic of the experimental setup. (B) Picture of (i) the microfluidic chip and resulting COMSOL simulations for (ii) $\mathrm{H}_{2} \mathrm{O}_{2}$ concentration profile at $\mathrm{t}=10$ min after stopping the flow, $(\mathrm{C})$ at different flow rates and (D) different time intervals after stop flow actuation. (E) The simulated $\mathrm{H}_{2} \mathrm{O}_{2}$ concentration gradients as a function of time .

\subsection{Chemotaxis assay}

The microswimmers used in this assay are silica based Janus particles half coated with copper, that were introduced recently. ${ }^{[20]}$ When these Janus particles are dispersed in $\mathrm{H}_{2} \mathrm{O}_{2}$ solution, the Cu cap catalyzes the $\mathrm{H}_{2} \mathrm{O}_{2}$ degradation on one side of the particle and therefore creates an unbalanced distribution of product ions, which leads to a fluid flow propelling the particle towards the copper cap direction (Figure 2 B). Based on the excellent agreement that we found between the behaviour of these particles in external flows and simulations using a self-electrophoretic mechanism in our previous work, we assume different catalytic activities between the equator and the pole of the copper cap that cause an electric field similar to $^{[21]}$ and build a finite numerical simulation model by COMSOL (Figure 2 D). 


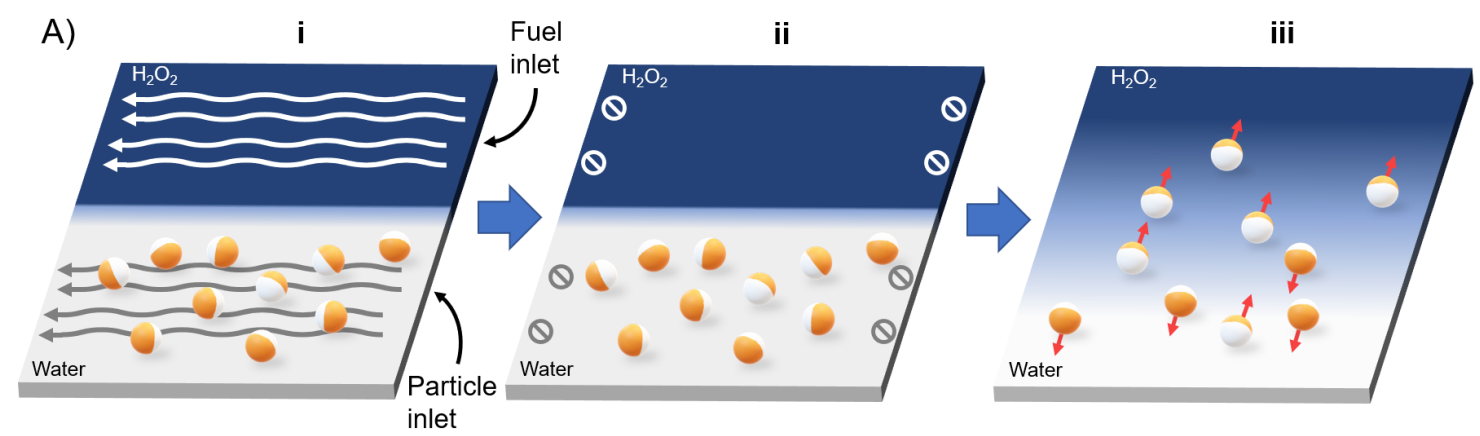

B)

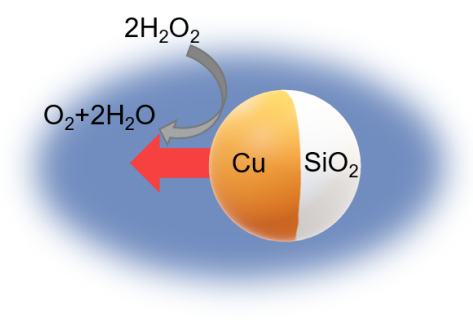

C)

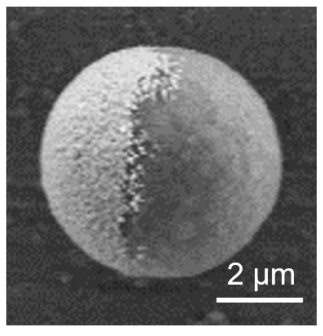

D)

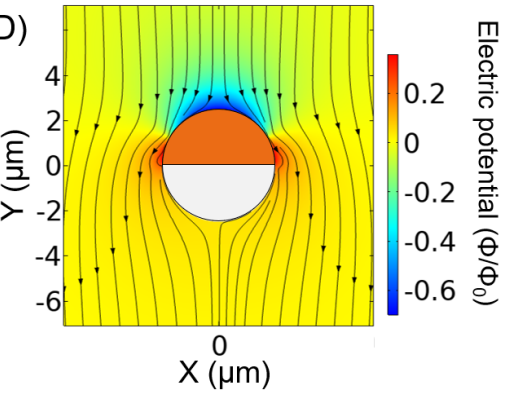

Figure 2: Intrinsic propulsion and chemotaxis of $\mathrm{Cu} @ \mathrm{SiO}_{2}$ micromotors. A) Operating principles of the positive and negative chemotaxis of $\mathrm{Cu} @ \mathrm{SiO}_{2}$ micromotors. B) Schematic diagram of the intrinsic propulsion of $\mathrm{Cu}_{\mathrm{SiO}} \mathrm{Sicromotors}_{2}$ in $\mathrm{H}_{2} \mathrm{O}_{2}$. C) Scanning electrical micrograph of a $\mathrm{Cu} @ \mathrm{SiO}_{2}$ microsphere. D) Electric potential (color coded) and flow field lines (black arrows) around a $\mathrm{Cu} @ \mathrm{SiO}_{2}$ Janus micromotors.

In the previously described microfluidic setup, two reservoirs containing water and a solution of $2,5 \%$ hydrogen peroxide were pressurized using an Elveflow pressure controller. The suspension of active particles was then injected with a syringe into the water streamline prior to the microfluidic chip. The two streams were then injected into the microfluidic chip at a constant flow rate until a stable gradient of $\mathrm{H}_{2} \mathrm{O}_{2}$ was established in the chamber. The flow was then stopped and videos were recorded in the field of view around the middle of the chamber, where the gradient was formed (Figure $2 \mathrm{~A}$ ). A control experiment was done by replacing the medium of the microswimmers (i.e. water) with hydrogen peroxide to remove any gradient but still keep the activity. Particles' trajectories were extracted from the recorded videos using a homemade tracking software in Matlab.

A number of selected particle trajectories are illustrated on Figure 3. The Cu cap of the Janus sphere is easily identified with brightfield microscopy and this allowed to recover the particles orientation. When plotting the cap orientation angle of at least 200 particles in the presence and absence of $\mathrm{H}_{2} \mathrm{O}_{2}$ gradient, a clear difference is noticeable. In the control experiment, the orientation of motion is isotropic, there is no preferred direction (Figure 3 A.iii and S3 A). On the other hand, in the presence of a gradient, a significant anisotropy is visible along the gradient (Figure 3 B.iii and S3 B). Indeed, the microswimmers are swimming strongly upward the gradient, towards the more concentrated area. There is also a clear, although slightly weaker, trend downward the gradient. 
A)
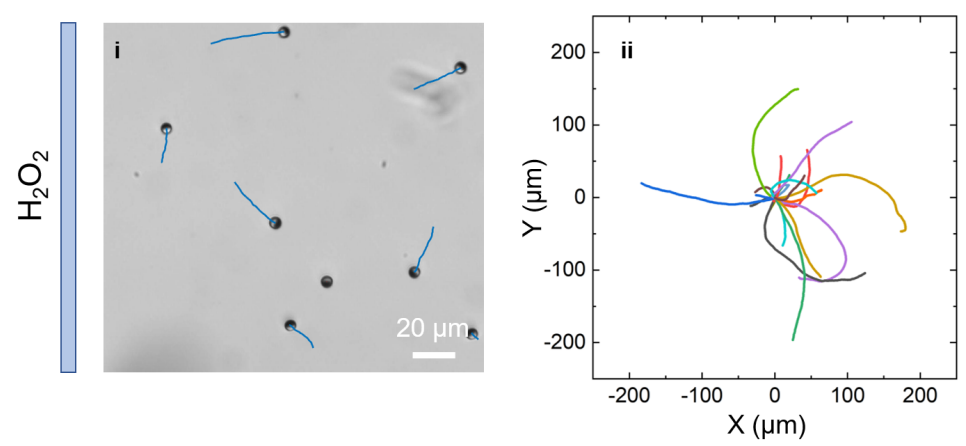

B)

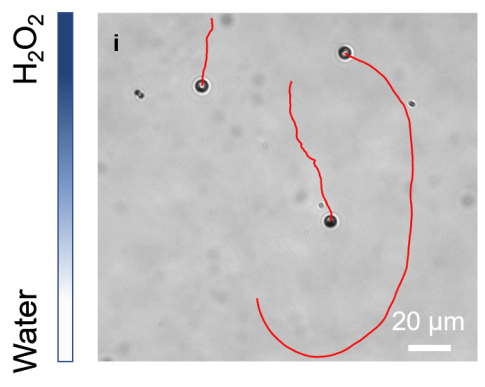

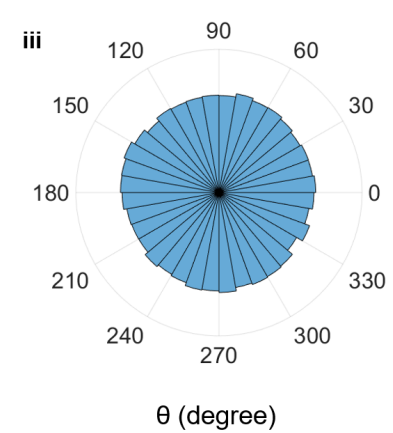
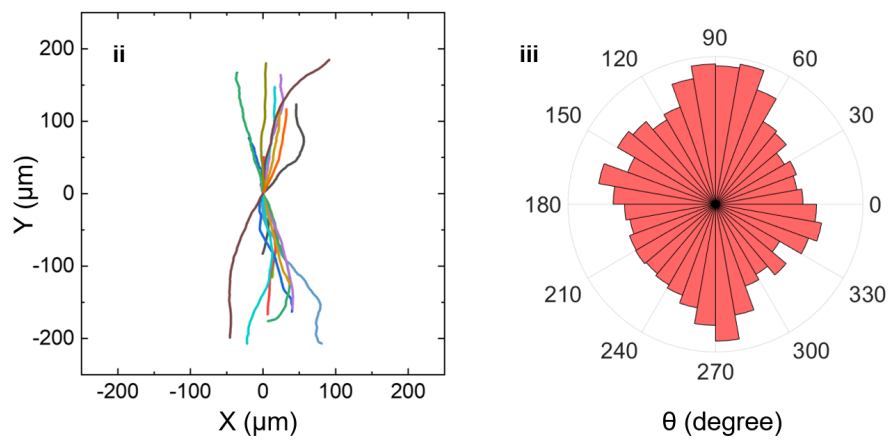

Figure 3: Statistical comparisons between chemotaxis and non-chemotaxis behaviors of $\mathrm{Cu} @ \mathrm{SiO}_{2}$ micromotors. (i) Optical

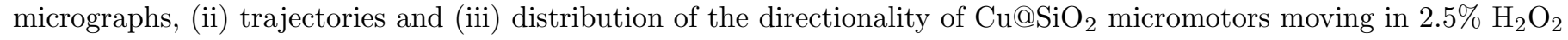
with no gradient (A) and the gradient (B).

\section{Discussion}

The upward motion of active Janus particles towards the region of more concentrated fuel, also called positive chemotaxis, was first described by Baraban et al. ${ }^{[13]}$ Using a flowing gradient, this study was not considering at all the (then unknown) potential cross-stream migration effect. ${ }^{[22]}$ Using a pressure driven setup and a feedback loop, we managed to decouple both effects and clearly observed the chemotactic response of these particles. As expected, we found a symmetry in the behavior with both positive and negative chemotaxis being present in the same set of experiments. To help understand our findings, numerical simulations with COMSOL were made to model the flow field around the microswimmers in different configurations (see Figure 4). Depending on their original orientation in the vicinity of the fuel gradient, the particles will experience forces and torques that will influence a change in their orientation. If the particles are initially oriented perpendicular to the flow, the cap facing the region more concentrated in peroxide, the chemical conversion is equal and symmetric on both sides of the cap; thus leading to a symmetric flow field around the particles and no forces changing their orientation apart from rotational diffusion. The same principles apply when the particles are oriented downward the gradient, the drag force exerted on them is uniform and does not induce any directional change thus explaining the great proportion of microswimmers swimming in this direction. 
A)
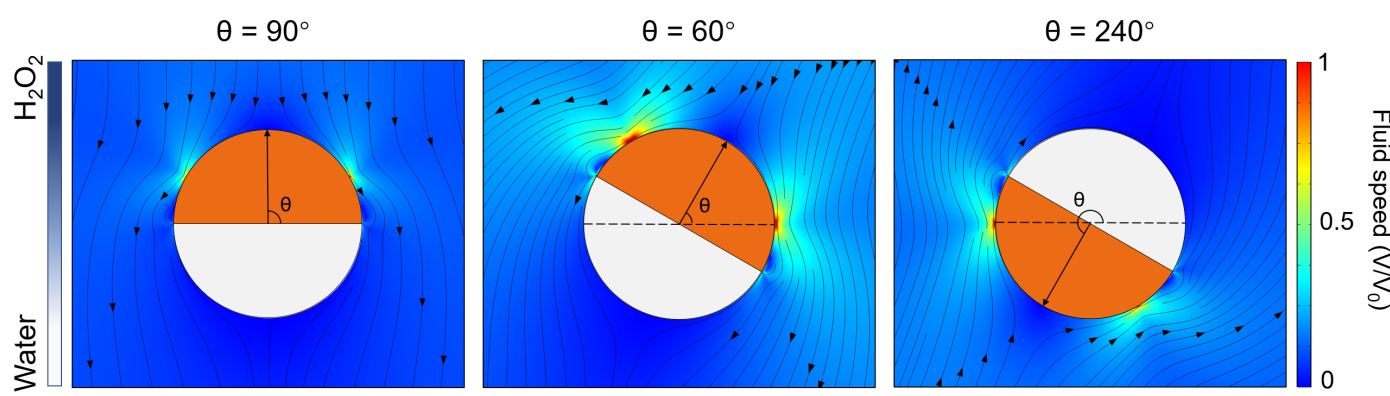

B)
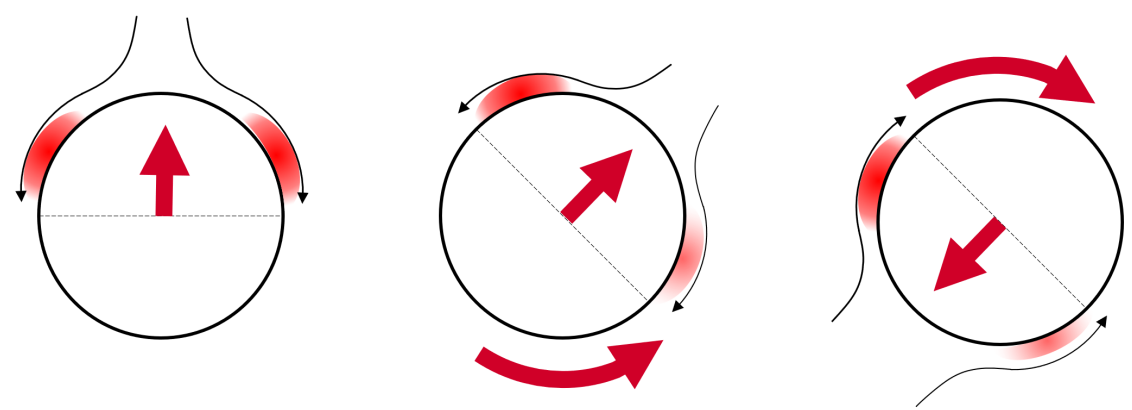

Figure 4: Finite element simulation of $\mathrm{Cu} @ \mathrm{SiO}_{2}$ micromotors in a chemical gradient with different orientation configurations. A) Fluid speed magnitude (color coded) and flow field lines (black arrows) around a $\mathrm{Cu} @ \mathrm{SiO}_{2}$ micromotor when the orientation of the particle aligns with the gradient $\left(\theta=90^{\circ}\right)$ or deviate from it $\left(\theta=60^{\circ}\right.$ and $\left.\theta=240^{\circ}\right)$. B) Schematic of the flow induced torque exerted on the particle forcing it to modify its orientation and align with the gradient direction.

However, this theory is insufficient to explain the results obtained and the clear asymmetry and higher proportion of particles oriented towards the 90 degree angle compared to the 270 degree angle. All particles in the gradient area that are not aligned parallel to the gradient will experience uneven drag forces. These forces will induce a torque that will reorient the particles' cap towards the 90 degree angle. The propulsion force being proportional to the amount of oxygen created during the chemical decomposition of $\mathrm{H}_{2} \mathrm{O}_{2}$, a higher concentration of fuel leads to a higher propulsion force and consequently, a higher drag force.

To verify this theory more explicitly, we analyze the moving direction changes during the chemotaxis behavior of particles (see Figure 5). By comparing the orientation of the Janus particles with the direction of motion, we confirm that both are correlated and that a change in orientation will induce a change in the velocity vector. Furthermore, we confirmed the underlying hypothesis that a change in orientation is immediately followed by a change in the velocity vector. Recent work have reported similar chemotaxis behavior by orientation rectification of $\mathrm{ZnO}$ micromotors. ${ }^{[23]}$

Initially, our results seem to contradict the predictions of Popescu et al. that depending on the contrast of surface mobilities, each Janus particle is characterized by a tendency to rotate either towards, or away from a chemical gradient. ${ }^{[2]}$ While it is difficult to a priori predict whether $\mathrm{Cu} @ \mathrm{SiO}_{2}$ particles belong to one group ('quadrant') that should orient to swim gradient up or the other, our experimental finding of nearly same occurrence of positive and negative chemotaxis, question the concept. However, a detailed study of different Janus particles ${ }^{[25,26,27]}$ or particles in different fuels ${ }^{[28]}$ is beyond the scope of this manuscript but is required to broaden our understanding before making such conjectures. 
A)

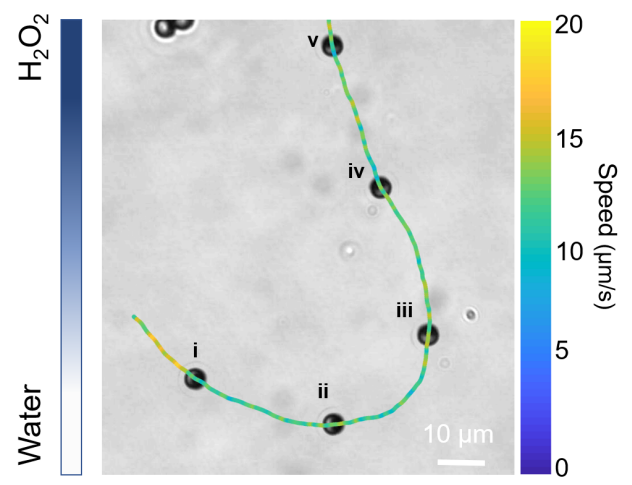

B)
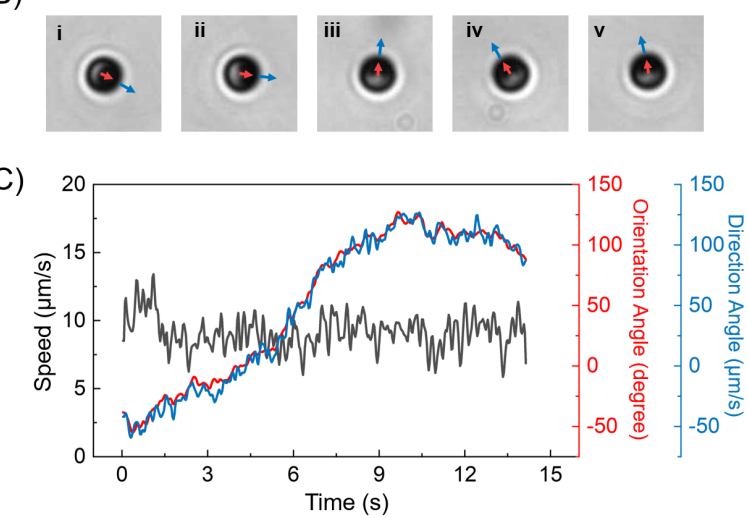

Figure 5: Orientation changes lead to apparent chemotaxis. (A) Representative trajectory of a $\mathrm{Cu}_{\mathrm{S}} \mathrm{SiO}_{2}$ micromotor changing its swimming direction to align with the gradient. (B) Direction (blue arrow) and orientation (red arrow) of the $\mathrm{Cu} @ \mathrm{SiO}_{2}$ micromotor in (A) at different time intervals. (C) Instantaneous speeds, direction angles and orientation angles of the the $\mathrm{Cu} @ \mathrm{SiO}_{2}$ micromotor in $(\mathrm{A})$.

\section{Conclusion}

Using pressure pumps to precisely control flow rates in a microfluidic gradient generation system, we successfully performed a fast stop-and-go flow experiment to study apparent chemotaxis of Janus microswimmers. We observed and studied the physical mechanisms behind the swimming pattern of opper coated silica microparticles in a gradient of hydrogen peroxide. By analyzing the orientation angle of the particles, a clear trend indicates both positive and negative chemotaxis. Studying the flow field surrounding the microswimmers using COMSOL simulations, we were able to describe the forces and torques applied on the particles when placed in a gradient as fuel, leading to a change in their orientation. Three main cases were identified: if the particles have their cap oriented towards the more concentrated region, no change in orientation is due to the gradient; if the cap is askew in the gradient, the particles will reorient its trajectory towards the high concentration; and finally, a particle oriented downward the gradient will not be affected by any torque and will keep its initial orientation. Further studies applying this technology to other types of microswimmers and fuels will be necessary to generalize the physical concepts.

\section{Supporting Information}

Supporting Information containing the experimental section, information on the numerical simulation, information on the supporting videos and additional information on established gradients and resulting angles, is available from the Wiley Online Library or from the author.

\section{Acknowledgements}

This project has received funding from the European Union's Horizon 2020 research and innovation program under the Marie Skłodowska-Curie grant agreement No 812780. J.S. and Z.X. acknowledge a Freigeist grant (no 91619) from the Volkswagen foundation and a Fulbright Cottrell Award, which partially supported this study. Z.X. acknowledges financial support from China Scholarship Council.

\section{Conflicts of Interest}

Two authors A.N. and B.G., at the time of this work, were employees of Elvesys SAS, a for profit company that sells Elveflow equipment, which was used for flow control and measurements in this work.

\section{References}

[1] B. Chance, Rev. Sci. Instrum. 1951, 22, 619-627. 
[2] C. R. Bagshaw in Stopped-Flow Techniques (Ed.: G. C. K. Roberts), Springer Berlin Heidelberg, Berlin, Heidelberg, 2013, pp. 2460-2466.

[3] D. Dendukuri, S. S. Gu, D. C. Pregibon, T. A. Hatton, P. S. Doyle, Lab Chip 2007, 7, 818-828.

[4] G. M. Whitesides, nature 2006, 442, 368-373.

[5] P. Sharan, A. Nsamela, S. C. Lesher-Pérez, J. Simmchen, Small 2021, 17, 2007403.

[6] V. Sourjik, N. S. Wingreen, Curr. Opin. Cell Biol. 2012, 24, 262-268.

[7] L. Stephens, L. Milne, P. Hawkins, Curr. Biol. 2008, 18, R485-R494.

[8] Y. Ji, X. Lin, Z. Wu, Y. Wu, W. Gao, Q. He, Angew. Chem. 2019, 131, 12328-12333.

[9] K. K. Dey, X. Zhao, B. M. Tansi, W. J. Méndez-Ortiz, U. M. Córdova-Figueroa, R. Golestanian, A. Sen, Nano lett. 2015, 15, 8311-8315.

[10] C. Lozano, C. Bechinger, Nat. commun. 2019, 10, 1-9.

[11] C. Lozano, B. Ten Hagen, H. Löwen, C. Bechinger, Nat. commun. 2016, 7, 1-10.

[12] L. Niese, L. Wang, S. Das, J. Simmchen, Soft Matter 2020, 16, 10585-10590.

[13] L. Baraban, S. M. Harazim, S. Sanchez, O. G. Schmidt, Angew. Chem. Int. Ed. 2013, 52, 55525556 .

[14] K. K. Dey, S. Bhandari, D. Bandyopadhyay, S. Basu, A. Chattopadhyay, Small 2013, 9, $1916-1920$.

[15] A. Somasundar, S. Ghosh, F. Mohajerani, L. N. Massenburg, T. Yang, P. S. Cremer, D. Velegol, A. Sen, Nat. Nanotechnol. 2019, 14, 1129-1134.

[16] S. Yu, Y. Cai, Z. Wu, Q. He, View 2021, 20200113.

[17] J. Katuri, K. Seo, D. Kim, S. Sanchez, Lab Chip 2016, 16, 1101-1105.

[18] S. Saha, R. Golestanian, S. Ramaswamy, Phys. Rev. E 2014, 89, 062316.

[19] J. Agudo-Canalejo, P. Illien, R. Golestanian, Nano lett. 2018, 18, 2711-2717.

[20] P. Sharan, Z. Xiao, V. Mancuso, W. E. Uspal, J. Simmchen, Upstream rheotaxis of catalytic Janus spheres, 2021, ChemRxiv preprint DOI: 10.26434/chemrxiv-2021-9s6wz.

[21] S. Ebbens, D. Gregory, G. Dunderdale, J. Howse, Y. Ibrahim, T. Liverpool, R. Golestanian, EPL (Europhysics Letters) 2014, 106, 58003.

[22] J. Katuri, W. E. Uspal, J. Simmchen, A. Miguel-López, S. Sánchez, Sci. Adv. 2018, 4, eaao1755.

[23] F. Mou, Q. Xie, J. Liu, S. Che, L. Bahmane, M. You, J. Guan, Natl. Sci. Rev. 2021, 8, nwab066.

[24] M. N. Popescu, W. E. Uspal, C. Bechinger, P. Fischer, Nano lett. 2018, 18, 5345-5349.

[25] D. P. Singh, W. E. Uspal, M. N. Popescu, L. G. Wilson, P. Fischer, Adv. Funct. Mater. 2018, 28, 1706660.

[26] J. Simmchen, J. Katuri, W. E. Uspal, M. N. Popescu, M. Tasinkevych, S. Sánchez, Nat. commun. 2016, 7, 1-9.

[27] A. T. Brown, I. D. Vladescu, A. Dawson, T. Vissers, J. Schwarz-Linek, J. S. Lintuvuori, W. C. Poon, Soft Matter 2016, 12, 131-140.

[28] L. Feuerstein, C. G. Biermann, Z. Xiao, C. Holm, J. Simmchen, J. Am. Chem. Soc. 2021, 143, 17015-17022. 
Table of Contents

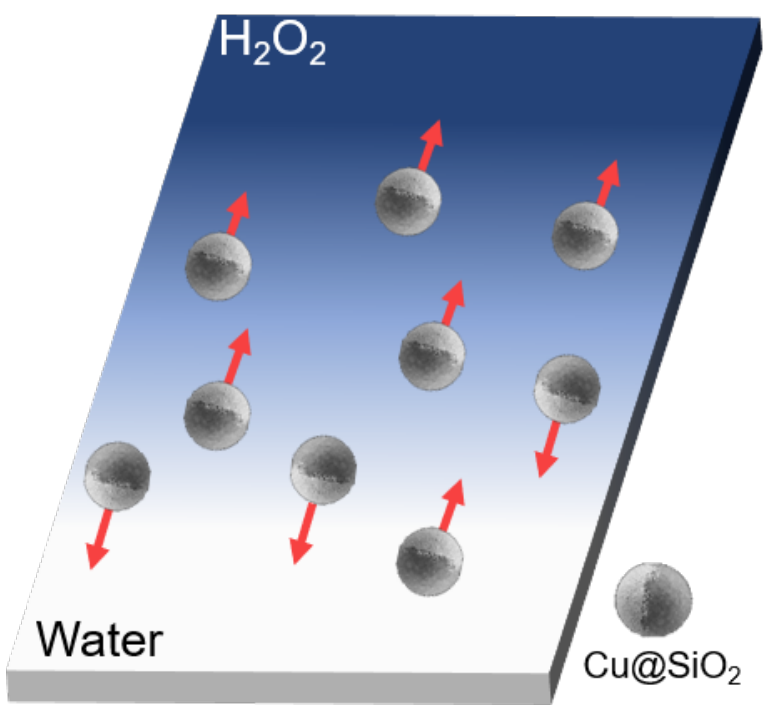

ToC Entry

Janus microswimmers show positive and negative chemotaxis behavior in a microfluidic assay. A flowbased linear gradient is generated and the flow is stopped during the chemotaxis assay using a retroactive pressure loop. 